\title{
OPEN Effects of sub-threshold transcutaneous auricular vagus nerve stimulation on cerebral blood flow
}

\author{
Conan Chen ${ }^{1 \bowtie}$, Yixiang Mao ${ }^{1}$, Maryam Falahpour ${ }^{1}$, Kelly H. MacNiven ${ }^{2,3}$, Gary Heit ${ }^{3,4}$, \\ Vivek Sharma ${ }^{3}$, Konstantinos Alataris ${ }^{3} \&$ Thomas T. Liv ${ }^{1 \bowtie}$
}

Transcutaneous auricular vagus nerve stimulation (taVNS) has shown promise as a non-invasive alternative to vagus nerve stimulation (VNS) with implantable devices, which has been used to treat drug-resistant epilepsy and treatment-resistant depression. Prior work has used functional MRI to investigate the brain response to taVNS, and more recent work has also demonstrated potential therapeutic effects of high-frequency sub-threshold taVNS in rheumatoid arthritis. However, no studies to date have measured the effects of high-frequency sub-threshold taVNS on cerebral blood flow (CBF). The objective of this study was to determine whether high-frequency $(20 \mathrm{kHz})$ subthreshold taVNS induces significant changes in CBF, a promising metric for the assessment of the sustained effects of taVNS. Arterial spin labeling (ASL) MRI scans were performed on 20 healthy subjects in a single-blind placebo-controlled repeated measures experimental design. The ASL scans were performed before and after $15 \mathrm{~min}$ of either sub-threshold taVNS treatment or a sham control. taVNS induced significant changes in CBF in the superior posterior cerebellum that were largely localized to bilateral Crus I and Crus II. Post hoc analyses showed that the changes were driven by a treatment-related decrease in CBF. Fifteen minutes of high-frequency sub-threshold taVNS can induce sustained CBF decreases in the bilateral posterior cerebellum in a cohort of healthy subjects. This study lays the foundation for future studies in clinical populations, and also supports the use of ASL measures of CBF for the assessment of the sustained effects of taVNS.

Vagus nerve stimulation (VNS) with implantable devices is currently used to treat drug-resistant epilepsy and treatment-resistant depression. However the cost and complications associated with implantation have limited the application of invasive VNS to a broader range of health conditions ${ }^{1-4}$ and spurred growing interest in non-invasive approaches, such as transcutaneous auricular vagus nerve stimulation (taVNS $)^{4-7}$. In taVNS, an electrode on the surface of the ear delivers a small electrical current across the skin to the auricular branch of the vagus nerve. Stimulation of the auricular branch in turn activates other areas of the brain through connected pathways $^{8,9}$, including regions involved in the regulation of the autonomic nervous system (ANS). The theory behind the therapeutic efficacy of taVNS is that stimulating the vagus nerve can correct maladaptive processing within the nervous system as well as neuro-immune communications that are associated with negative health outcomes. Building upon prior work that suggests a link between ANS dysfunction and inflammation ${ }^{10}$, a recent open-label study of subjects with rheumatoid arthritis demonstrated clinically relevant changes in both disease biomarkers and symptoms after 12 weeks of sub-threshold taVNS treatment ${ }^{11}$. The therapeutic benefits of taVNS have also been reported in the context of depression ${ }^{5,10,12}$ and migraine ${ }^{13}$, with mounting evidence linking these clinical effects to neural responses ${ }^{4,6,14}$.

While the mechanisms through which taVNS causes changes are not completely understood, a number of recent studies have used blood oxygen-level dependent (BOLD) functional MRI (fMRI) to characterize the acute brain response to taVNS ${ }^{1,4,6,14-16}$. While BOLD fMRI is an effective approach for measuring evoked brain responses, its susceptibility to low-frequency noise sources makes it less effective for measuring changes that

${ }^{1}$ Center for Functional MRI, Department of Radiology, University of California San Diego, 9500 Gilman Drive \#0677, La Jolla, CA 92093, USA. ²Department of Psychology, Stanford University, Stanford, CA, USA. ${ }^{3}$ Nēsos Corporation, Redwood City, CA, USA. "Department of Neurosurgery, Hue University of Medicine and Pharmacy, Hue, Vietnam. ${ }^{\circledR}$ email: coc004@health.ucsd.edu; ttliu@health.ucsd.edu 


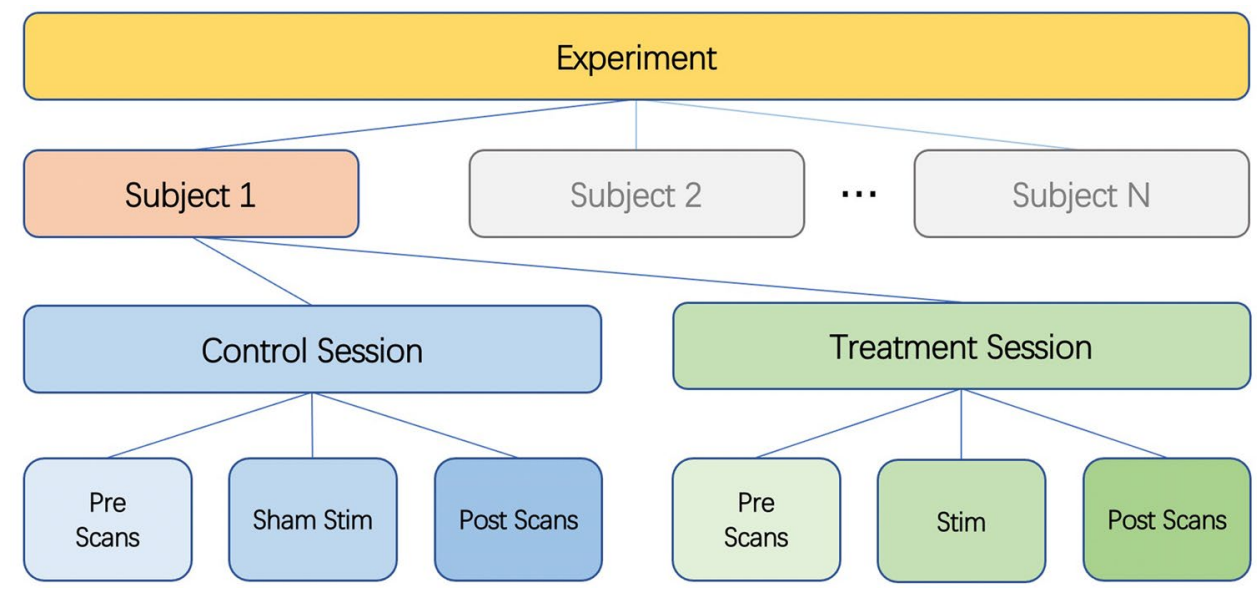

Figure 1. Diagram of experimental design. Each subject undergoes a control session and a treatment session. Each session consists of pre scans, a stimulation (sham for control session) section, and post scans.

occur with time periods greater than several minutes ${ }^{17}$. In contrast, measures of cerebral blood flow (CBF), a well-defined physiological quantity, can be used to characterize changes in brain physiology occurring over periods ranging from seconds to years ${ }^{18}$, and therefore constitute a promising metric for the assessment of the sustained effects of VNS.

In prior work, both SPECT and PET have been used to characterize the CBF response to VNS applied using implantable devices in patients with either major depression (MD) or epilepsy. In MD patients, SPECT has been used to demonstrate regionally dependent increases and decreases in CBF due to 4 to 10 weeks of chronic VNS ${ }^{19,20}$. In a PET study with MD patients, Conway et al..$^{21,22}$ reported regionally dependent increases and decreases in the evoked CBF response to short duration (90 s) VNS. For epilepsy patients, Henry et al. ${ }^{23,24}$ reported PET-based CBF increases and decreases in the evoked CBF reponse to $30 \mathrm{~s}$ of VNS, with the effects diminishing after 12 weeks of chronic VNS. In addition, two earlier pilot studies ${ }^{25,26}$ reported VNS evoked changes in PET CBF measures.

To the best of our knowledge, no studies to date have examined the CBF response to taVNS. In this study, we used arterial spin labeling (ASL) MRI to measure changes in CBF induced by $15 \mathrm{~min}$ of taVNS in healthy subjects, employing a high-frequency $20 \mathrm{kHz}$ stimulus similar to that employed by Marsal et al. ${ }^{11}$. In contrast to SPECT and PET, ASL MRI does not require the injection of radioactive tracers, making it especially suitable for repeated measures in a healthy population ${ }^{27,28}$. Furthermore, in this study the taVNS is applied at a sub-threshold level, such that subjects cannot readily determine if they are receiving the treatment or a placebo. In contrast to prior studies where the perceptible sensations associated with VNS may have complicated the interpretation of the results, the sub-threshold stimulus used in this study removes somatosensory confounds and is therefore compatible with a single-blind placebo-controlled design. The goal of this study was to determine whether a single course of taVNS with duration similar to that of the daily dose employed in ${ }^{11}$ could lead to detectable changes in CBF.

\section{Material and methods}

Overview. We used a single-blind placebo-controlled experimental design. Twenty seven healthy subjects were enrolled in this study after providing informed consent (14 females and 13 males, aged 19-40 years). The study was approved by the UCSD Institutional Review Board (IRB), and informed consent was obtained from all participants. All experiments were performed in accordance with relevant guidelines and regulations. Figure 1 shows the overall experiment structure. Each subject participated in a control session and a treatment session, with the order randomized across subjects. The sessions were separated by at least one day to avoid taVNS carryover effects.

All scanning visits consisted of a pre scan section, a stimulation (or sham) section, and a post scan section. For the first 6 subjects, the subjects wore the stimulation earpieces throughout the entire session, and the stimulation section took place while they remained lying inside the scanner. To improve the subject experience, the protocol was slightly modified for the subsequent 21 subjects. These 21 subjects wore regular earplugs during the MRI scans, came out of the scanner for the stimulation section, and then returned to the scanner with regular earplugs to finish the post scan section.

MRI scans. Scans were acquired on a GE Discovery MR750 3.0 T system. Pre and post scan sections consisted of (1) a high-resolution anatomical scan (MPRAGE, resolution $=1 \mathrm{~mm}^{3}, 208$ slices, FOV $=25.6 \mathrm{~cm}$, $\mathrm{TR}=2500 \mathrm{~ms}, \mathrm{TE}=2.92 \mathrm{~ms}, \mathrm{FA}=8 \mathrm{deg}$ ), (2) resting-state BOLD fMRI scans, and (3) arterial spin labeling (ASL) scans (2D pseudo-continuous ASL (PCASL), single-shot spiral, 100 repetitions, resolution $=3.75 \mathrm{~mm} \times 3.75 \mathrm{~mm}$, slice thickness $=6 \mathrm{~mm}$, matrix size $=64 \times 64 \times 24$, FOV $=24 \mathrm{~cm}, \mathrm{TE}=3.2 \mathrm{~ms}$, TR $=4300 \mathrm{~ms}$, labeling duration $=1800 \mathrm{~ms}$ and post-labeling delay $=1800 \mathrm{~ms})^{28}$. Only the anatomical and ASL scans are analyzed here. The resting-state BOLD scans will be analyzed in a separate paper. During all ASL scans, subjects rested with their eyes open and gently focused on a fixation point. 
taVNS. taVNS was delivered using custom-fit ear pieces designed by Nēsos ${ }^{\circledR}$ (Redwood City, CA). A bipolar configuration was used and two circular (radius $0.3 \mathrm{~cm}$ ) hydrogel electrodes were placed on the ear piece surface interfacing with the cymba concha, with a contact area of $0.28 \mathrm{~cm}^{2}$ for each electrode.

Alcohol wipes were used to clean the cymba concha prior to inserting the ear pieces. Stimulation was applied to the left ear, targeting vagal afferents in the cymba concha based on known vagus nerve anatomy ${ }^{8,29}$. For all visits, the stimulation (or sham) section took place between the pre and post scan sections.

On both scan days, a sensation test was performed to determine a subject's threshold for perceiving the stimulation, which entailed stimulating at $1.0 \mathrm{~mA}$ and increasing by increments of $0.5 \mathrm{~mA}$ (up to a maximum of $5.0 \mathrm{~mA}$ ). Criteria for perceiving the stimulation include sensations of stinging, pricking, or warmth. This procedure was repeated until the subject reported feeling the stimulation at the same level three times. The sensation test was followed by a 15 -min stimulation period. The stimulation was delivered continuously at $20 \mathrm{kHz}$, using biphasic square waves with pulse width of 20 us. The stimulation amplitude was set at $75 \%$ of a subject's perceptual threshold (1.5-3.8 mA; impedance 3-9.5 k $\Omega$ at $20 \mathrm{kHz}$ ) on the "active" stimulation day and was set to zero on the control day ("sham" condition). The order of "active" and "sham" stimulation days was randomized across subjects. To verify that the subthreshold stimulation did not cause any local heating, we used a DTT-1372 Thermometer (Ames Instruments ${ }_{\mathrm{TM}}$, Harbor Freight Tools USA. Inc., Calabasas, CA) to measure temperature changes over a 15-min period in both the active and sham conditions for one of the subjects. The probe was placed on the medial surface of the external ear, directly across from the earpiece that was positioned at the cymba concha on the lateral surface of the external ear. Minimal temperature increases were observed for active $\left(0.20^{\circ} \mathrm{C}\right)$ and sham $\left(0.25^{\circ} \mathrm{C}\right)$ conditions with no significant difference $(\mathrm{p}=0.46)$ in the slope of the changes over the 15 -min period.

Since the stimulation was delivered at $75 \%$ of perceptual threshold, subjects were blinded to whether they were undergoing the sham or stimulus treatment. At the end of their second session, all subjects were asked whether they could identify their control and treatment sessions across their two visits.

Data processing and analysis. Data from the ASL scan were volume registered and quantified into physiological CBF units $(\mathrm{mL} / 100 \mathrm{~g} / \mathrm{min})$ via local tissue correction with a proton-density scan acquired during the same scan section ${ }^{30}$. The SCORE algorithm ${ }^{31}$ was used on a per-slice basis to censor outlier timepoints in the $\mathrm{CBF}$ time series before computing the average CBF map. Using AFNI software ${ }^{32}$, the high-resolution anatomical scans were transformed into MNI space, and the CBF volumes were then warped to MNI space using the transformation matrices from the anatomical transformation, with a final voxel resolution of $4 \mathrm{~mm}^{3}$.

For group analysis in MNI space, Post minus Pre CBF difference maps (termed "Post-Pre" for the remainder of the manuscript) were computed for control and treatment sessions. A two-sided paired t-test was performed for the main contrast $(\text { Post-Pre })_{\text {treatment }}-(\text { Post-Pre })_{\text {control }}$ on a per-voxel basis using AFNI $3 \mathrm{dttest}++$. This contrast is equivalent to the interaction effect for a two-way (Post, Pre by Treatment, Control) repeated measures ANOVA. After setting a primary voxelwise threshold, spatial clustering thresholds were used to control the family-wise error rate (FWER) associated with detecting clusters of supra-threshold t-values across the 27,168 voxels in the MNI brain mask $^{33}$. For a specified voxelwise threshold, a permutation-based method (as implemented in AFNI $3 \mathrm{dttest++}$ with the -Clustsim option) was used to estimate the false positive rates associated with detecting clusters with varying cluster-size thresholds. Additional details and background on this approach are provided in Cox et al. $2017^{33}$. A voxelwise threshold of $\mathrm{p}<0.01$ and a cluster size threshold of 22 voxels (clusters defined with voxel faces touching) were used to assess results at a FWE-corrected $\mathrm{p}<0.05$ level.

Post-hoc two-sided paired t-tests were also performed for each session (control and treatment), and were assessed at a voxelwise threshold $\mathrm{p}<0.01$ and a FWE-corrected $\mathrm{p}<0.05$ level (using cluster size thresholds of 35 and 51 voxels for the control and treatment contrasts, respectively). In addition, post-hoc analyses were performed using the mean CBF values of any significant clusters found from the main contrast of (Post-Pre) treatment $_{\text {t }}$ - (Post-Pre) control $_{\text {. }}$

Finally, subjects that were randomized to a session order of control followed by treatment were compared to subjects randomized to treatment then control. A post-hoc two-sided two-sample t-test was performed between these two groups using the mean CBF values of any significant clusters found from the main contrast.

\section{Results}

Participants and stimulation. Out of twenty-seven total subjects, two dropped out after their first session, due in part to the COVID-19 related suspension of research activities during the course of the study. The remaining twenty-five subjects completed both the control and treatment sessions. Five subjects were excluded from the study due to technical errors or severe image artifacts in at least one of their CBF scans, leaving twenty total subjects included in the analysis. These twenty subjects received a mean stimulation amplitude of $3.24 \pm 0.56 \mathrm{~mA}$ $(\min =1.5 \mathrm{~mA}, \max =3.8 \mathrm{~mA})$. The control and treatment sessions were matched to take place at the same time each day whenever possible, with an average time-of-day difference of $0.9 \pm 1.32 \mathrm{~h}(\min =0 \mathrm{~h}, \max =4 \mathrm{~h})$.

When asked whether they could identify the control and treatment sessions across their two visits, sixteen of the twenty subjects responded that they could not guess. Of the remaining four subjects who indicated they could identify their sessions, three guessed correctly and one guessed incorrectly.

Overall, an average of $24.81 \pm 6.93$ min elapsed from the end of the stimulation to the start of the post section ASL scan for these subjects.

Group-level whole-brain analysis. For the main contrast shown in Fig. 2a, application of clustering analysis at voxelwise $\mathrm{p}<0.01$ and FWE-corrected $\mathrm{p}<0.05$ revealed one significant cluster spanning both hemispheres of the posterior cerebellum (71 voxels, peak MNI coordinate $[-6,78,-36])$. Figure $2 b, c$ show session-level con- 


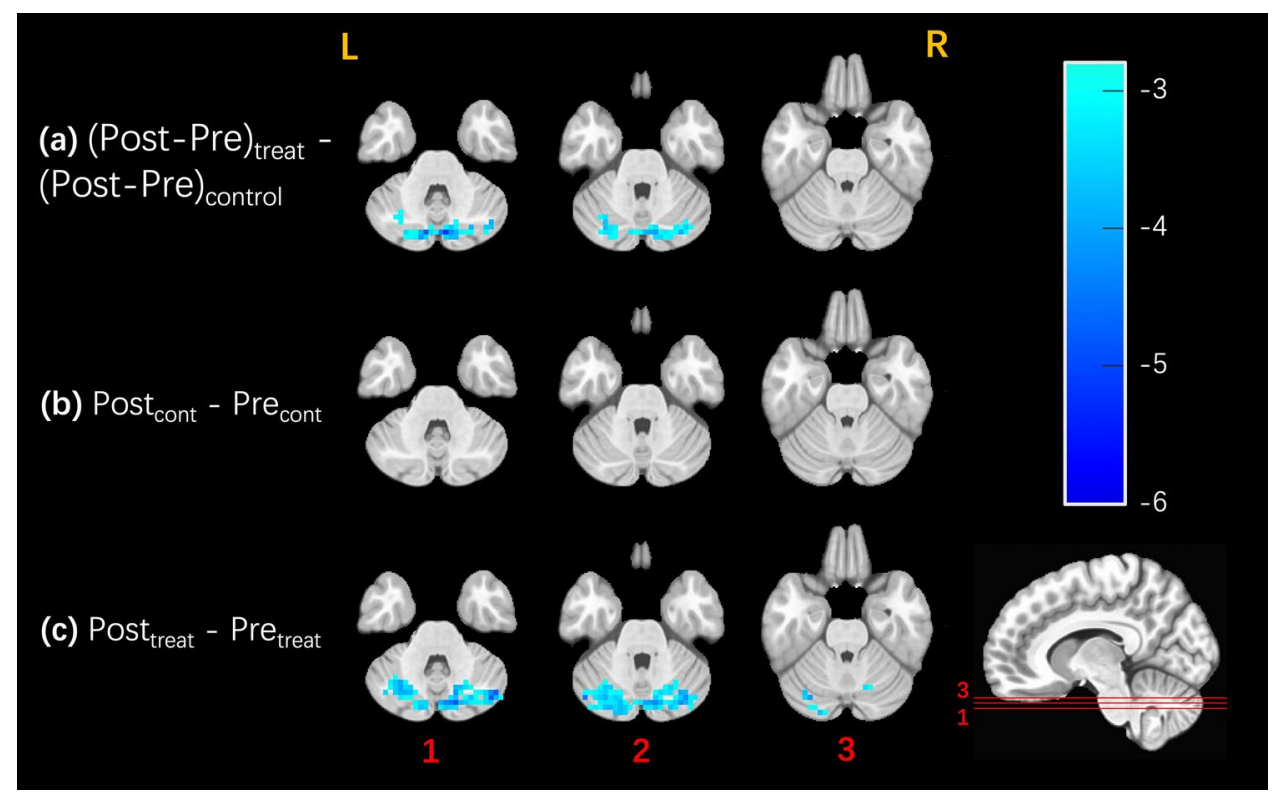

Figure 2. Maps showing clusters of voxels with significant effects, FWE-corrected at $\mathrm{p}<0.05$ with voxelwise threshold $\mathrm{p}<0.01$. Contrast-dependent cluster size thresholds based on permutation testing are indicated below. Surviving voxels are color-coded by their t-statistic value and overlaid on anatomical images, with the same slices shown for all three contrasts. No significant clusters were observed in any of the other slices. (a) For the main (Post-Pre) $)_{\text {treatment }}-(\text { Post-Pre) })_{\text {control }}$ contrast , one cluster with 71 voxels survived with a cluster size threshold of 22 voxels. (b) For the Post control $_{-}$Pre $_{\text {control }}$ contrast, no clusters survived with a cluster size threshold of 35 voxels. (c) For the Post treatment - Pre $_{\text {treatment }}$ contrast, two clusters survived with a cluster size threshold of 51 voxels.

trasts that support the main cluster result. Figure $2 \mathrm{~b}$ shows no significant CBF changes in the control session, while Fig. 2c shows a left hemispheric cerebellar cluster (69 voxels) and a right hemispheric cerebellar cluster (74 voxels) with significant CBF decreases in the treatment session.

We used the probabilistic atlas of the cerebellar lobules as defined in $^{34,35}$ and the AFNI whereami function to determine the overlap of the significant clusters with the cerebellar lobules. For the main contrast, the single cluster showed $96.3 \%$ overlap with the cerebellar lobules, with $47.2 \%$ of the cluster overlapping with left and right Crus I, $44.8 \%$ with left and right Crus II, 3.5\% with Vermis Crus II, and $0.8 \%$ with left and right lobules VIIb. For the treatment contrast, the left hemispheric cluster had 94\% overlap with the cerebellar lobules, with $75.5 \%$ overlap with left Crus I, $15.8 \%$ with left Crus II, and 2.6\% with left lobule VI. The right hemispheric cluster had $89.7 \%$ overall overlap consisting of $45.4 \%$ overlap with right Crus I, $28.5 \%$ with right Crus II, $8 \%$ with right lobule VI, $3.3 \%$ with right dentate, $2.7 \%$ with Vermis Crus II, $1.8 \%$ with left Crus II, and $0.1 \%$ with right VIIb.

Post-hoc analyses of the mean CBF values from the main contrast cluster are shown in Fig. 3. The cluster shows a significant decrease in CBF related to treatment $(\mathrm{p}<0.001,-15.90 \%)$ alongside a statistically insignificant increase in the control session $(\mathrm{p}=0.19,+4.02 \%)$. Consistent with the maps shown in Fig. 2 , the post-hoc analyses indicate that the contrast of interest mainly reflects significant treatment-related decreases in cerebellar CBF.

Post-hoc analysis of session-ordering effects in the main cluster is shown in Fig. 4. There was not a significant difference in the main contrast values between the two session orders $(p=0.54)$, indicating that the main contrast effect is not driven by either randomization order.

\section{Discussion}

Our findings reveal a significant bilateral decrease in cerebellar CBF as a result of 15 min of sub-threshold taVNS, reflecting physiological changes that persisted for many minutes after the treatment. These effects were observed using a stimulus paradigm and duration similar to those of the daily dose employed in the recent open label clinical trial of ${ }^{11}$.

In contrast to the current findings, prior studies have reported both unilateral ${ }^{26}$ and bilateral ${ }^{21,23,24}$ increases in cerebellar CBF. However, there are several key methodological differences that are worth noting. First and foremost, the prior studies examined evoked CBF changes in response to the concurrent application of 30 to $90 \mathrm{~s}$ of VNS, whereas the current study assessed resting-state CBF measures obtained before and after the application of taVNS. Thus, the prior findings are limited to characterizing the acute cerebellar CBF response to VNS, while the current findings offer evidence of a sustained decrease in cerebellar CBF in reponse to taVNS. Second, VNS consists of an implantable device targeting the cervical vagus nerve, while taVNS is non-invasive and applied to a branch originating from the superior ganglion of the vagus nerve. This difference in neural targets may be expected to produce different physiological responses. Third, the use of a sub-threshold high frequency $(20 \mathrm{kHz})$ 


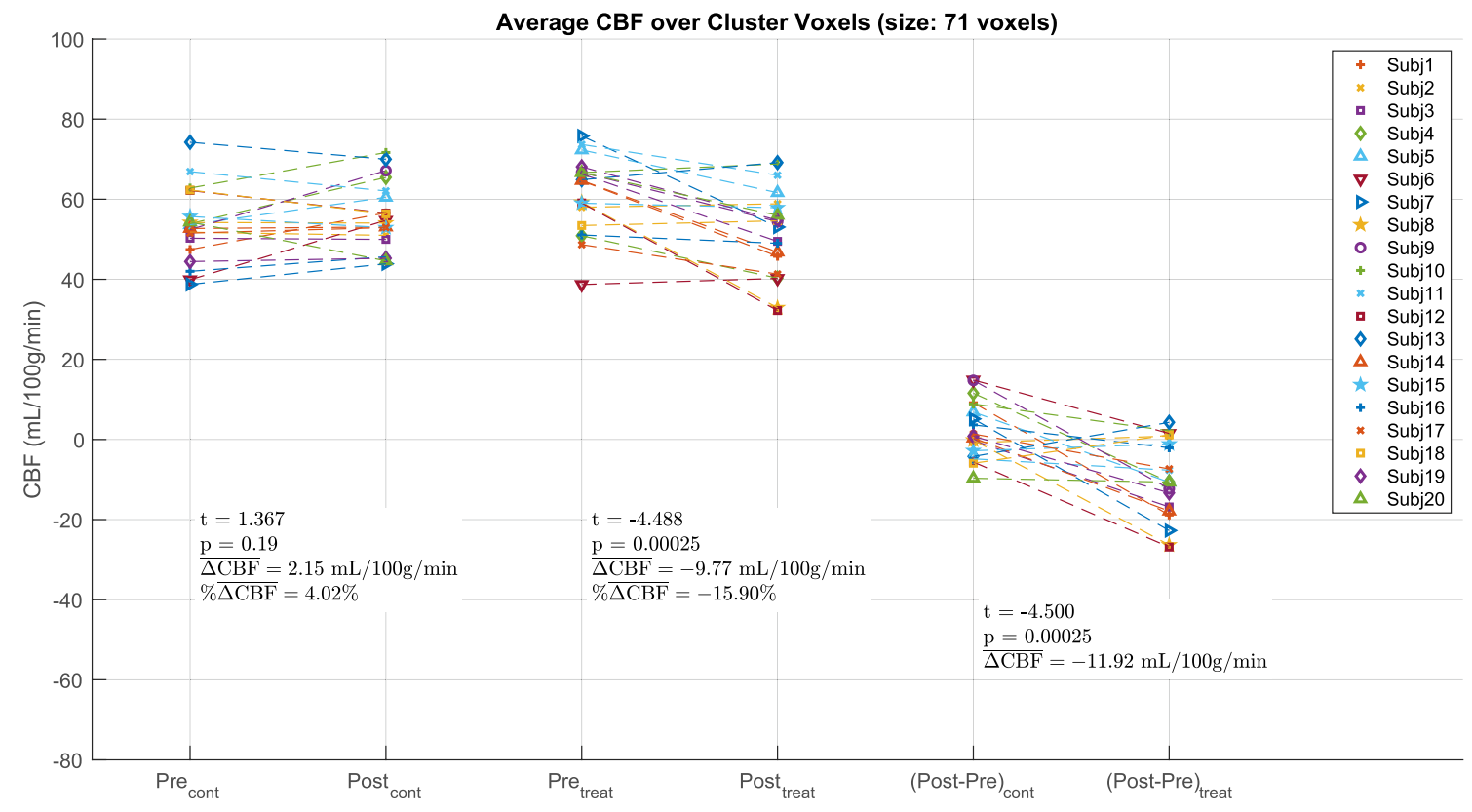

Figure 3. Post-hoc analysis with mean per-subject CBF values from the cluster shown in Fig. 2a. The Pre and Post values from the control and treatment sessions are shown in the leftmost and center pairs, respectively. The (Post-Pre) CBF differences from the control and treatments sessions are shown in the rightmost pair. There was a significant treatment-related decrease in $\mathrm{CBF}(\mathrm{p}<0.001,-15.90 \%)$ alongside a weak, statistically insignificant increase in the control session $(\mathrm{p}=0.19,+4.02 \%)$, resulting in a significant main effect $(\mathrm{p}<0.001)$.

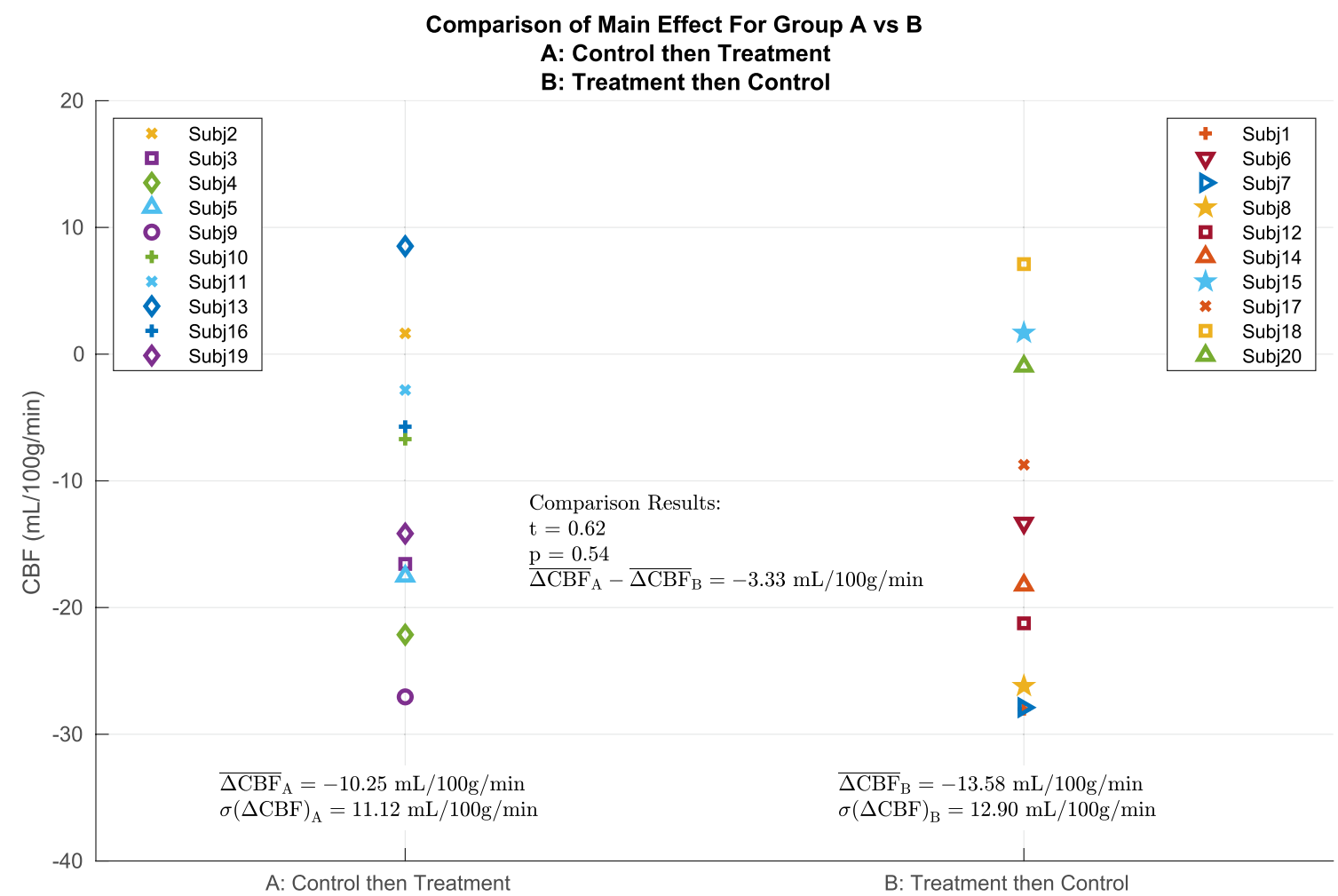

Figure 4. The main effect for any significant differences in effect between the subjects randomized to A: Control then Treatment vs B: Treatment then Control. The plot below shows the main contrast values of (Post-Pre) $_{\text {treatment }}-(\text { Post-Pre) })_{\text {control }}$ within the main contrast cluster reported in Fig. 3. On the left are subjects randomized to group $\mathrm{A}$; on the right are subjects randomized to group B. A two-sided two-sample t-test was performed between the two groups, indicating no significant difference in main effect $(p=0.54)$ between the two session orders. 
stimulus in the current study enabled the use of a single-blind placebo controlled design, in contrast to the openlabel design with the super-threshold low frequency $(20$ to $30 \mathrm{~Hz})$ stimuli employed in prior studies. The placebo control in the current study enabled a clean isolation of the direct vagal stimulation effects from those associated with stimulus-associated sensations and responses, a separation that was not possible with prior designs. In addition, there are likely to be frequency dependent neurophysiological mechanisms that lead to differences in the effects between the high $(20 \mathrm{kHz})$ versus low $(20$ to $30 \mathrm{~Hz})$ frequency stimulus paradigms. Finally, the current findings were obtained in healthy subjects who were naïve to taVNS, whereas the prior results characterized the responses in MD or epilepsy patients at a time point that was hours to months after the commencement of continuous VNS therapy. Thus, the current study provides critical information about the effect of taVNS on the healthy brain that can serve as a baseline for the future assessment of effects in clinical populations.

Stimulus-induced changes in cerebellar activity have also been observed with BOLD fMRI, but the experimental methods and results greatly vary across studies with reports of both evoked activations in healthy subjects ${ }^{1,15,16}$ and epileptic patients ${ }^{36}$ and deactivations in healthy subjects ${ }^{2}$ and MD subjects ${ }^{37,38}$. As the BOLD signal exhibits a complex dependence on neural, vascular, and metabolic factors, a definitive link between observed BOLD changes and underlying CBF changes cannot be established without additional experimental measures ${ }^{39}$. However for most conditions, task-evoked increases and decreases in the BOLD signal are generally thought to reflect concomitant CBF increases and decreases. Thus, while the fMRI findings cannot resolve whether VNS induces increases or decreases in cerebellar CBF, they offer general support for the potential of VNS to modulate CBF levels in the cerebellum.

In this study, significant CBF changes were limited to the cerebellum. The prior PET and SPECT studies reported $\mathrm{CBF}$ changes in additional brain regions, although the results were highly variable across studies. As noted above, a number of these studies considered the evoked CBF response as compared to the sustained response reported here. Two SPECT studies examined the effects of 4 to 10 weeks of chronic VNS ${ }^{19,20}$ and reported increases and decreases in $\mathrm{CBF}$ in multiple brain regions, but there were differences between the two studies in the regions that were identified and neither study reported cerebellar CBF changes. In light of the variability observed in prior studies and the significant methodological differences discussed above, discrepancies between the prior and current findings are to be expected. In particular, the interpretation of the changes observed in the prior studies typically discussed the potential modulation of activity in regions that were thought to be associated with the disease state. These brain regions would not necessarily show an effect in healthy subjects. Nevertheless, a future study examining the effects of sub-threshold taVNS over a longer period (e.g. weeks) would be of interest to determine if $\mathrm{CBF}$ changes can be detected in areas beyond the cerebellum.

While the cerebellum has traditionally been viewed as a motor control region, a wealth of evidence suggests that it also plays important roles in cognitive, affective, and social processing ${ }^{40-42}$. In particular, the cerebellar regions Crus I and Crus II, which exhibited the greatest overlap with significant CBF changes in this study, are considered to be primarily associated with non-motor functions, such as working memory, executive function, language processing, social mentalizing, and emotional cognition ${ }^{41,43,44}$. Interestingly, the cerebellum has a purported role in conditions for which taVNS has beneficial effects. For example, cerebellar dysfunction has been implicated in major depressive disorder $(\mathrm{MDD})^{45}$, and a preliminary clinical study found that a multi-week regime of taVNS (relative to sham) can alleviate depression severity as assessed via the Hamilton Depression Scale in MDD patients ${ }^{5}$. TaVNS-induced cerebellar activation has also been reported in an fMRI study of MDD patients, though individual differences in activation were not related to clinical outcomes ${ }^{46}$. Furthermore, taVNS has shown promise as a treatment for migraine ${ }^{13}$, another disorder that has been linked to functional and structural alterations within the posterior cerebellum, namely Crus I and Crus $\mathrm{II}^{47,48}$. Finally, a recent study found that a 12-week regime of taVNS treatment (with parameters similar to those used in this study) improved symptoms for patients with rheumatoid arthritis (RA) ${ }^{11}$. Reduced gray matter of the cerebellum has been associated with higher levels of peripheral inflammation in RA patients ${ }^{49}$. Together, these findings suggest that the link between taVNS therapeutic efficacy and cerebellar function should be further explored.

There are limitations to the study that would be important to address in future follow-up work. First, the analysis required that all four ASL scans $\left(\right.$ Pre $_{\text {control }}$, Post $_{\text {control }}$, Pre $_{\text {treatment }}$, and Post $\left.t_{\text {treatment }}\right)$ were free from artifacts, as issues in any run could affect the main contrast. However, this strict approach makes the study sensitive to any issues like subject motion that can cause artifacts in the CBF maps, leading us to exclude five out of twentyfive subjects, even though a much lower fraction (10 out of 100) of the scans exhibited artifacts. For future work using ASL to probe taVNS effects, one improvement would be to use ASL sequences with higher SNR, such as 3D PCASL with background suppression to reduce motion sensitivity ${ }^{50,51}$. The increased SNR can be used to shorten the scan lengths while maintaining similar image quality, mitigating the risk of motion associated with longer scan times. The higher SNR also increases the ability to detect CBF changes in a group analysis, so that fewer subjects would be needed to detect the same effects, increasing the study robustness to subject exclusions. Second, there is a small possibility of a thermo-regulatory vascular confound in response to local energy deposition from taVNS. We believe this to be unlikely, as no subjects reported sensations of heating at the stimulation site. Furthermore, an in-situ thermal test of the device found minimal $\left(<0.3^{\circ} \mathrm{C}\right)$ changes in temperature over a 15 -min period in both active and sham conditions. However, we cannot completely rule out a vascular response that quickly dissipates heat and could prevent any detection of warmth despite an underlying change in neural response.

In this study, the taVNS was applied at the cymba concha, which is $100 \%$ innervated by the auricular branch of the vagus nerve ${ }^{8}$. However, given the overlapping innervation by the greater auricular nerve and the auriculotemporal nerve of auricular regions proximal to the cymba concha ${ }^{8}$, it is not possible to completely rule out the effects on CBF through these pathways. For example, the auriculotemporal nerve lies within the mandibular branch of the trigeminal nerve, and prior work indicates that stimulation of the trigeminal nerve can cause changes in $\mathrm{CBF}^{52}$. 
In conclusion, the present results indicate that $15 \mathrm{~min}$ of high-frequency sub-threshold taVNS can induce sustained CBF decreases in the bilateral posterior cerebellum in a sample of healthy subjects. Further work is needed to determine if similar changes can be observed in clinical populations and to assess if the measures can provide greater insight into the mechanisms underlying treatment outcomes. In addition, future research should also address how stimulation parameters such as frequency, duration, and electrode location impact the response.

Received: 22 July 2021; Accepted: 1 December 2021

Published online: 15 December 2021

\section{References}

1. Badran, B. W. et al. Neurophysiologic effects of transcutaneous auricular vagus nerve stimulation (taVNS) via electrical stimulation of the tragus: A concurrent taVNS/fMRI study and review. Brain Stimul. 11, 492-500 (2018).

2. Dietrich, S. et al. A novel transcutaneous vagus nerve stimulation leads to brainstem and cerebral activations measured by functional MRI. Biomed. Tech. 53, 104-111 (2008).

3. Kraus, T. et al. BOLD fMRI deactivation of limbic and temporal brain structures and mood enhancing effect by transcutaneous vagus nerve stimulation. J. Neural Transm. 114, 1485-1493 (2007).

4. Fang, J. et al. Transcutaneous vagus nerve stimulation modulates default mode network in major depressive disorder. Biol. Psychiatry 79, 266-273 (2016).

5. Rong, P. et al. Effect of transcutaneous auricular vagus nerve stimulation on major depressive disorder: a nonrandomized controlled pilot study. J. Affect. Disord. 195, 172-179 (2016).

6. Garcia, R. G. et al. Modulation of brainstem activity and connectivity by respiratory-gated auricular vagal afferent nerve stimulation in migraine patients. Pain 158, 1461-1472 (2017).

7. Addorisio, M. E. et al. Investigational treatment of rheumatoid arthritis with a vibrotactile device applied to the external ear. Bioelectron. Med. 5, 4 (2019).

8. Peuker, E. T. \& Filler, T. J. The nerve supply of the human auricle. Clin. Anat. 15, 35-37 (2002).

9. Kaniusas, E. et al. Current directions in the auricular vagus nerve stimulation I-A physiological perspective. Front. Neurosci. 13, 854 (2019).

10. Liu, C. H. et al. Neural networks and the anti-inflammatory effect of transcutaneous auricular vagus nerve stimulation in depression. J. Neuroinflamm. 17, 1-11 (2020).

11. Marsal, S. et al. Non-invasive vagus nerve stimulation for rheumatoid arthritis: a proof-of-concept study. Lancet Rheumatol. 3, E262-E269 (2021).

12. Kong, J., Fang, J., Park, J., Li, S. \& Rong, P. Treating depression with transcutaneous auricular vagus nerve stimulation: State of the art and future perspectives. Front. Psychiatry 9, 1-8 (2018).

13. Straube, A., Ellrich, J., Eren, O., Blum, B. \& Ruscheweyh, R. Treatment of chronic migraine with transcutaneous stimulation of the auricular branch of the vagal nerve (auricular t-VNS): A randomized, monocentric clinical trial. J. Headache Pain 16, 1-9 (2015).

14. Zhang, Y. et al. Transcutaneous auricular vagus nerve stimulation at $1 \mathrm{~Hz}$ modulates locus coeruleus activity and resting state functional connectivity in patients with migraine: An fMRI study. NeuroImage Clin. 24, 101971 (2019).

15. Frangos, E., Ellrich, J. \& Komisaruk, B. R. Non-invasive access to the vagus nerve central projections via electrical stimulation of the external ear: FMRI evidence in humans. Brain Stimul. 8, 624-636 (2015).

16. Yakunina, N., Kim, S. S. \& Nam, E. C. Optimization of transcutaneous vagus nerve stimulation using functional MRI. Neuromodulation 20, 290-300 (2017).

17. Wang, J. et al. Arterial spin labeling perfusion fMRI with very low task frequency. Magn. Reson. Med. 49, 796-802 (2003).

18. Liu, T. T. \& Brown, G. G. Measurement of cerebral perfusion with arterial spin labeling: Part 1. Methods. J. Int. Neuropsychol. Soc. 13, 517-525 (2007).

19. Zobel, A. et al. Changes in regional cerebral blood flow by therapeutic vagus nerve stimulation in depression: An exploratory approach. Psychiatry Res. Neuroimaging 139, 165-179 (2005).

20. Kosel, M., Brockmann, H., Frick, C., Zobel, A. \& Schlaepfer, T. E. Chronic vagus nerve stimulation for treatment-resistant depression increases regional cerebral blood flow in the dorsolateral prefrontal cortex. Psychiatry Res. Neuroimaging 191, 153-159 (2011).

21. Conway, C. R. et al. Cerebral blood flow changes during vagus nerve stimulation for depression. Psychiatry Res. Neuroimaging 146, 179-184 (2006).

22. Conway, C. R. et al. Brain blood-flow change with acute vagus nerve stimulation in treatment-refractory major depressive disorder. Brain Stimul. 5, 163-171 (2012).

23. Henry, T. R. et al. Brain blood flow alterations induced by therapeutic vagus nerve stimulation in partial epilepsy: I. Acute effects at high and low levels of stimulation. Epilepsia 39, 983-990 (1998).

24. Henry, T. R., Bakay, A. E., Pennell, P. B., Epstein, C. M. \& Votaw, R. Brain blood-flow alterations induced by therapeutic vagus nerve stimulation in partial epilepsy: II. Prolonged effects at high and low levels of stimulation. Epilepsia 45, 1064-1070 (2004).

25. Garnett, E. S., Nahmias, C., Scheffel, A., Firnau, G. \& Upton, A. R. M. Regional cerebral blood flow in man manipulated by direct vagal stimulation. Pacing Clin. Electrophysiol. 15, 1579 (1992).

26. Ko, D. et al. Vagus nerve stimulation activates central nervous system structures in epileptic patients during PET H2150 blood flow imaging. Neurosurgery 39, 426-430 (1996).

27. Detre, J. A., Rao, H., Wang, D. J. J., Chen, Y. F. \& Wang, Z. Applications of arterial spin labeled MRI in the brain. J. Magn. Reson. Imaging 35, 1026-1037 (2012).

28. Alsop, D. C. et al. Recommended implementation of arterial spin-labeled perfusion MRI for clinical applications: A consensus of the ISMRM Perfusion Study Group and the European Consortium for ASL in Dementia. Magn. Reson. Med. 73, 102-116 (2015).

29. Berthoud, H.-R. \& Neuhuber, W. L. Functional and chemical anatomy of the afferent vagal system. Auton. Neurosci. Basic Clin. 85, 1-17 (2000).

30. Shin, D. D., Ozyurt, I. B. \& Liu, T. T. The cerebral blood flow biomedical informatics research network (CBFBIRN) database and analysis pipeline for arterial spin labeling MRI data. Front. Neuroinform. 7, 21 (2013).

31. Dolui, S., Wang, Z., Shinohara, R. T., Wolk, D. A. \& Detre, J. A. Structural correlation-based outlier rejection (SCORE) algorithm for arterial spin labeling time series. J. Magn. Reson. Imaging 45, 1786-1797 (2017).

32. Cox, R. W. AFNI: Software for analysis and visualization of functional magnetic resonance neuroimages. Comput. Biomed. Res. 29, 162-173 (1996).

33. Cox, R. W., Chen, G., Glen, D. R., Reynolds, R. C. \& Taylor, P. A. FMRI clustering in AFNI: false-positive rates redux. Brain Connect. 7, 152-171 (2017).

34. Diedrichsen, J., Balsters, J. H., Flavell, J., Cussans, E. \& Ramnani, N. A probabilistic MR atlas of the human cerebellum. Neuroimage 46, 39-46 (2009).

35. Diedrichsen, J. et al. Imaging the deep cerebellar nuclei: A probabilistic atlas and normalization procedure. Neuroimage 54, 1786-1794 (2011). 
36. Liu, W.-C., Mosier, K., Kalnin, A. J. \& Marks, D. BOLD fMRI activation induced by vagus nerve stimulation in seizure patients. J. Neurol. Neurosurg. Psychiatry 74, 811-813 (2003).

37. $\mathrm{Mu}$, Q. et al. Acute vagus nerve stimulation using different pulse widths produces varying brain effects. Biol. Psychiatry 55, 816-825 (2004).

38. Nahas, Z. et al. Serial vagus nerve stimulation functional MRI in treatment-resistant depression. Neuropsychopharmacology 32, 1649-1660 (2007).

39. Liu, T. T., Glover, G. H., Mueller, B. A., Greve, D. N. \& Brown, G. G. An introduction to normalization and calibration methods in functional MRI. Psychometrika 78, 308-321 (2013).

40. Guell, X. \& Schmahmann, J. Cerebellar functional anatomy: a didactic summary based on human fMRI evidence. Cerebellum 19, $1-5(2020)$.

41. Van Overwalle, F. et al. Consensus Paper: Cerebellum and Social Cognition. Cerebellum. Vol. 19. (The Cerebellum, 2020).

42. Schmahmann, J. D. The cerebellum and cognition. Neurosci. Lett. 688, 62-75 (2019).

43. Guell, X., Gabrieli, J. D. E. \& Schmahmann, J. D. Triple representation of language, working memory, social and emotion processing in the cerebellum: convergent evidence from task and seed-based resting-state fMRI analyses in a single large cohort. Neuroimage 172, 437-449 (2018).

44. Stoodley, C. J. \& Schmahmann, J. D. Functional Topography of the Human Cerebellum. Handbook of Clinical Neurology. Vol. 154. (Elsevier B.V., 2018).

45. Depping, M. S., Schmitgen, M. M., Kubera, K. M. \& Wolf, R. C. Cerebellar contributions to major depression. Front. Psychiatry 9, 1-5 (2018).

46. Fang, J. et al. Early cortical biomarkers of longitudinal transcutaneous vagus nerve stimulation treatment success in depression. NeuroImage Clin. 14, 105-111 (2017).

47. Mehnert, J. \& May, A. Functional and structural alterations in the migraine cerebellum. J. Cereb. Blood Flow Metab. 39, 730-739 (2019).

48. Kros, L., Angueyra Aristizábal, C. A. \& Khodakhah, K. Cerebellar involvement in migraine. Cephalalgia 38, 1782-1791 (2018).

49. Schrepf, A. et al. A multi-modal MRI study of the central response to inflammation in rheumatoid arthritis. Nat. Commun. 9, 1-11 (2018).

50. Dolui, S. et al. Comparison of PASL, PCASL, and background-suppressed 3D PCASL in mild cognitive impairment. Hum. Brain Mapp. 38, 5260-5273 (2017).

51. Vidorreta, M. et al. Comparison of 2D and 3D single-shot ASL perfusion fMRI sequences. Neuroimage https://doi.org/10.1016/ Fj.neuroimage.2012.10.087 (2013).

52. White, T. G. et al. Trigeminal nerve control of cerebral blood flow: A brief review. Front. Neurosci. 15, 1-9 (2021).

\section{Acknowledgements}

This work was supported by a research grant from Nēsos Corporation, Redwood City, CA.

\section{Author contributions}

G.H., K.M., M.F., and T.L. designed the experiment. C.C. and T.L. wrote the main manuscript text. C.C. performed the processing and analysis of data and prepared all figures. Y.M., C.C. and M.F. collected the data used in the study. V.S. provided hardware support for the study. G.H., K.M., K.A. and T.L. provided interpretation of the data. All authors reviewed the manuscript.

\section{Competing interests}

Funding for this study was provided by an unrestricted grant from Nēsos (Redwood City, CA), which also provided hardware support for this study. Kelly H. MacNiven and Gary Heit report consultant fees from Nēsos. Konstantinos Alataris, Gary Heit and Vivek Sharma report equity holdings in Nēsos. Konstantinos Alataris and Vivek Sharma are current employees. Thomas Liu reports grants from Nēsos. All other authors declare no competing interests.

\section{Additional information}

Correspondence and requests for materials should be addressed to C.C. or T.T.L.

Reprints and permissions information is available at www.nature.com/reprints.

Publisher's note Springer Nature remains neutral with regard to jurisdictional claims in published maps and institutional affiliations.

(c) (i) Open Access This article is licensed under a Creative Commons Attribution 4.0 International cc) License, which permits use, sharing, adaptation, distribution and reproduction in any medium or format, as long as you give appropriate credit to the original author(s) and the source, provide a link to the Creative Commons licence, and indicate if changes were made. The images or other third party material in this article are included in the article's Creative Commons licence, unless indicated otherwise in a credit line to the material. If material is not included in the article's Creative Commons licence and your intended use is not permitted by statutory regulation or exceeds the permitted use, you will need to obtain permission directly from the copyright holder. To view a copy of this licence, visit http://creativecommons.org/licenses/by/4.0/.

(c) The Author(s) 2021 